\title{
Clinicopathologic Factors Associated with Mismatch Repair Status Among Filipino Patients with Young-Onset Colorectal Cancer
}

This article was published in the following Dove Press journal: Cancer Management and Research

\author{
Dennis Lee Sacdalan $\mathbb{D}^{1-3}$ \\ Reynaldo L Garcia ${ }^{2}$ \\ Michele H Diwa ${ }^{4}$ \\ Danielle Benedict Sacdalan (DD ${ }^{1,5}$ \\ 'Division of Medical Oncology, \\ Department of Medicine, University of \\ the Philippines, Manila, Philippines; \\ ${ }^{2}$ National Institute of Molecular Biology \\ and Biotechnology, University of the \\ Philippines, Diliman, Quezon City, \\ Philippines; ${ }^{3}$ Augusto P. Sarmiento \\ Cancer Institute, The Medical City, Pasig \\ City, Philippines; ${ }^{4}$ Department of \\ Pathology, University of the Philippines, \\ Manila, Philippines; ${ }^{5}$ Department of \\ Pharmacology and Toxicology, University \\ of the Philippines, Manila, Philippines
}

Introduction: Young-onset colorectal cancer is recognized as a distinct disease that may be sporadic or hereditary in nature. Microsatellite instability testing is recommended as a routine procedure in evaluating colorectal cancer specimens, especially in young-onset disease, because of implications in management. Immunohistochemistry of mismatch repair proteins serves as an inexpensive alternative to microsatellite instability testing with the added advantage of monitoring protein expression levels that may suggest underlying genetic or epigenetic alterations. This descriptive study aimed to determine the frequencies of proficient and deficient mismatch repair status among Filipino young-onset colorectal cancer patients, and to investigate their clinicopathologic profile.

Methods: Tumor tissues were prospectively collected from patients from two tertiary hospitals in the Philippines. Patients of age $\leq 45$ years with resected adenocarcinoma of the colon or rectum were recruited.

Results: Seventy-seven out of 124 patients had tumor samples sent for immunohistochemistry. Of these, 61 samples (79\%) were found to have proficient status while 16 samples $(21 \%)$ had deficient status. Mismatch repair protein deficiencies, when present, more commonly involved MSH2 and MSH6 (9\%) rather than MLH1 and PMS2 (5\%). The deficient group had a mean age of 37.1 years and a female preponderance $(56.25 \%)$, presenting as locally advanced ascending or descending colon tumors with mucinous histology in half of the population. The mismatch repair proficient group presented as locally advanced rectal and sigmoid tumors but with fewer mucinous adenocarcinomas $(26.2 \%)$ compared to the deficient group. In both the mismatch repair proficient and deficient patients with family history reports, most did not have any known relative with cancer $(75.4 \%$ and $68.75 \%$, respectively).

Conclusion: This is the first attempt to perform mismatch repair testing among young-onset colorectal cancer patients in the Philippines and to gather data on their clinicopathologic characteristics. However, the limited sample size precludes conclusive results for the associations of mismatch repair with clinicopathologic features.

Keywords: biomarkers, early-onset colorectal cancer, immunohistochemistry, microsatellite instability

\section{Introduction}

Colorectal cancer (CRC) ranks third among all incident cancers. ${ }^{1}$ In the Philippines, the number of new CRC cases has escalated from 5787 in 2010 to 9625 in 2015. ${ }^{2,3}$ Among individuals $<40$ years of age, new cases of CRC comprise $17-24 \%{ }^{4,5}$ The increasing incidence of this disease among young Filipinos mirrors recent findings reported in the United States. $^{6}$
Division of Medical Oncology, Department of Medicine, University of the PhilippinesPhilippine General Hospital, Taft Avenue,

Manila, 1000, Philippines

$\mathrm{Tel} / \mathrm{Fax}+63285263775$

Email dlsacdalan I@up.edu.ph
Cancer Management and Research 2021:13 2105-2II5 
CRC can develop through various mechanisms such as microsatellite instability (MSI), chromosomal instability, and aberrant methylation of $\mathrm{CpG}$ islands (CIMP) in the promoter regions of tumor suppressor genes. Notably, overlap between these mechanisms may also occur. ${ }^{7-10}$

The development of CRC in the young may be associated with hereditary conditions. Hereditary non-polyposis colorectal cancer (HNPCC) is the most common inherited cause of susceptibility to CRC. HNPCC is associated with germline mutations in DNA mismatch repair (MMR) genes, namely: MLH1, MSH2, MSH6, PMS2 and EPCAM. MMR-deficient (dMMR) tumors display high levels of microsatellite instability (MSI-H) in more than $97 \%$ of the time. ${ }^{11-13}$

Microsatellites are segments of DNA that contain tandem repeats, one to six bases long, that occur within the genome. Their numbers are ideally conserved within every human cell. Replication errors and defective mismatch repair result in MSI. For MSI to become detectable by conventional techniques, a significant number of cells, as in tumors, need to carry these abnormal microsatellites. Importantly, both hereditary and sporadic CRC can display MSI. ${ }^{12}$

An alternative to PCR-based testing is immunohistochemistry (IHC). The four-antibody panel involving MLH1, MSH2, MSH6 and PMS2 is used to determine MMR protein expression. Loss of at least one of these proteins, as demonstrated by lack of nuclear staining, indicates dMMR/MSI-H status. ${ }^{9}$

In the repair of DNA mismatches, MSH2 binds to MSH6 to form the heterodimer MutS $\alpha$, which is responsible for mismatch recognition and initiation of repair. ${ }^{14}$ After binding to DNA carrying a base mismatch, MutS $\alpha$ recruits the heterodimer MutL $\alpha$ composed of MLH1 and PMS2 in order to carry out the repair. Both MSH2 and MLH1 are obligatory partners that stabilize MSH6 and PMS2, respectively, from proteolytic degradation. ${ }^{15}$ Hence, in dMMR/MSI-H status, loss of MSH2 is expected to be accompanied by loss of MSH6 while loss of MLH1 comes with loss of PMS2. However, deficiency in MSH6 or PMS2 may occur even if MSH2 or MLH1 is intact due to compensatory binding of other MMR proteins to MSH2 or MLH1.

Pathologic findings that are predictive of MSI-H include: increased numbers of tumor infiltrating lymphocytes, lack of dirty necrosis and note of a Crohn's-like lymphoid reaction in the tumor tissue, any mucinous differentiation of the tumor, and age $<50$ at onset. ${ }^{16,17}$ There is controversy regarding the "sidedness" of these tumors. ${ }^{17-19}$
The dMMR/MSI-H status of CRC tumors has prognostic significance in several clinical scenarios. Young-onset $\mathrm{CRC}$ that is MSI-H is associated with more aggressive features such as mucinous and signet ring histology. ${ }^{20}$ In stage II disease, MSI-H tumors have excellent prognosis and do not benefit from adjuvant chemotherapy with 5fluorouracil. ${ }^{10}$ In contrast, MSI-H tumors in stage IV disease carry a poor prognosis but may show remarkable and durable responses to immune checkpoint inhibitors likely due to the rich immune response present within these tumors. $^{21,22}$ These observations highlight the importance of the early detection of dMMR/MSI-H CRCs.

Notably, $16 \%$ of young-onset CRC cases carry germline cancer susceptibility genes, thus the remaining population may be sporadic cases. The question then is whether young-onset $\mathrm{CRC}$ is an inherited or a sporadic disease. This issue is complicated by still poorly understood environmental risk exposures and by unidentified susceptibility genes or epigenetic mechanisms. ${ }^{23}$ Regardless of the underlying mechanism involved, it is vital to determine if a young-onset CRC tumor carries a dMMR/MSI-H phenotype because of the possible implications on management.

Due to the absence of local data, this study aims to determine the frequency of dMMR and MMR-proficient (pMMR) tumors among the recruited Filipino patients with young-onset CRC from two tertiary hospitals based on IHC. This technique is more advantageous compared to PCR-testing because of several reasons. First, IHC costs comparatively less than MSI testing. Second, it is more convenient since it necessitates resources that are already available in most pathology laboratories. Third, IHC can pinpoint MMR genes likely to be mutated. ${ }^{12}$

A second objective of the study is to compare the clinicopathologic profiles of dMMR and pMMR tumors from young CRC patients. This can provide a better understanding of the nature of young-onset CRC among Filipinos.

\section{Methods}

\section{Study Design and Patient Recruitment}

This study was performed on patients who were diagnosed with adenocarcinoma of the colon or rectum at age $\leq 45$ at the Philippine General Hospital (PGH) and The Medical City. Patients were enrolled regardless of stage of disease for as long as they were able to provide formalin-fixed, paraffin-embedded (FFPE) specimens adequate for IHC. 
Excluded were patients diagnosed with polyposis and inflammatory bowel disease due to alternative mechanisms involved in the pathogenesis of CRC.

\section{Specimen Collection and Preparation for IHC}

Specimens were obtained from surgically resected primary or metastatic tumors. Standard procedures for FFPE specimen preparation were done in the Surgical Pathology laboratory in PGH. Histological confirmation of the diagnosis of adenocarcinoma was performed by a pathologist on the hematoxylin-eosin stained sections. From the paraffin block, $3 \mu \mathrm{m}$-thick slices of tissue were cut and mounted on Dako silanized slides for IHC. The tissue was then incubated in a $60^{\circ} \mathrm{C}$ oven for one hour. Target retrieval solution $\left(\mathrm{pH}\right.$ 9.0) was then added at $97^{\circ} \mathrm{C}$ for fifteen minutes and then rinsed with wash buffer for five minutes.

\section{Immunohistochemical Staining for MMR Proteins}

Staining was performed on the four MMR proteins: MLH1, MSH2, MSH6 and PMS2. The IHC process was performed using the Dako Autostainer Link 48 and was carried out according to the manufacturer's guidelines. After staining, the slides were viewed under light microscopy. Brownish nuclear staining of weak to strong intensity in any number of tumor cells was considered a positive result.

\section{Validation Studies for IHC}

Prior to IHC staining of the subject specimens, validation studies were made to ensure the assay would perform as intended. Selected archival specimens of five known cases of sporadic colorectal adenocarcinoma were processed for IHC. Positive controls were derived from the validation slides and used for comparison with subject specimens. These positive controls demonstrated strong brown nuclear staining for all four antibodies against MMR proteins.

\section{Statistical Analysis}

Absolute and relative frequencies of dMMR and pMMR tumors were presented. Crude odds ratios were estimated to determine association between MMR status and clinicopathologic features including age, sex, number of relatives with cancer, disease stage, location of primary tumor, tumor grade, and histologic variants. Multiple logistic regression was done to generate adjusted odds ratios. All estimates were done at $95 \%$ confidence level.

\section{Ethical Considerations}

The study was conducted in accordance with the Declaration of Helsinki. The protocol was approved by the University of the Philippines Manila Research Ethics Board and The Medical City Institutional Review Board. All subjects gave their informed consent for inclusion before they participated in the study. Specifically, consent to retrieve tumor tissue specimens, extract demographical and clinicopathologic data, and follow-up survival status was obtained.

\section{Results}

A total of 124 patients aged 22 to 45 years old were recruited between May 2016 and August 2019. From these patients, 98 were able to turn over one FFPE sample each. Twenty-six patients with unretrieved samples were mainly those who underwent surgery in hospitals other than PGH or TMC, and those whose surgeries were deferred in favor of systemic chemotherapy due to disease progression. Out of the 98 tissue blocks retrieved, IHC for MMR protein expression was performed in 77 samples. IHC was not performed on 47 samples for various reasons concerning quality and quantity. Two were found to be second primary tumors and were thus excluded from the study. Complete pathologic response was noted in 7 resected specimens subjected to neoadjuvant chemoradiation. Twelve samples were deemed inadequate for IHC (Figure 1).

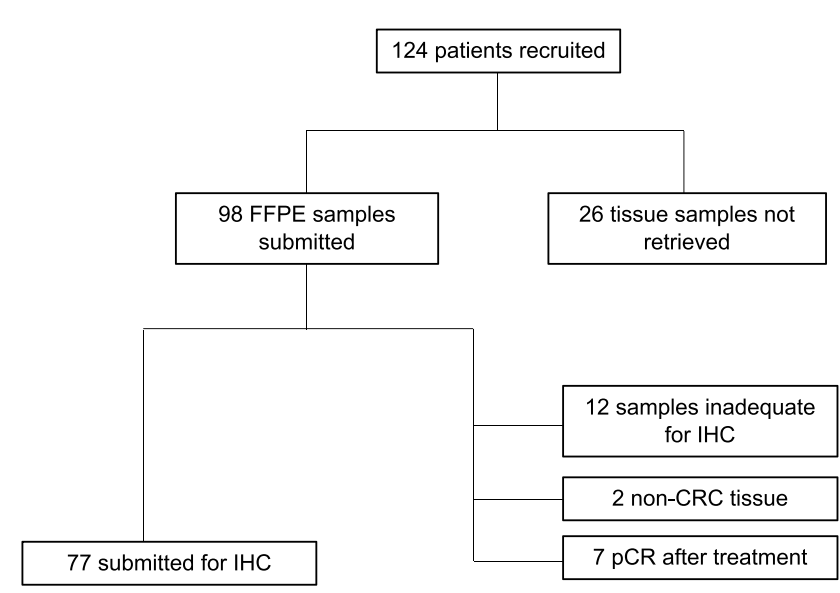

Figure I Flowchart of patient recruitment and tissue sample retrieval. Abbreviations: FFPE, formalin-fixed paraffin-embedded; IHC, immunohistochemistry; CRC, colorectal cancer; PCR, pathologic complete response. 


\section{Clinicopathologic Profile of Young-Onset CRC}

There were slightly more males than females $(52.4 \%$ vs $47.6 \%$, respectively). The mean age was 37.1 years; the youngest was 22 years old while the oldest was 45 years old. More patients (46.8\%) presented with locally advanced disease (stage III). Seventy six percent of patients had left-sided tumors (descending colon, sigmoid colon and rectum), most of which were in the rectum (45.2\%). There was one patient who presented with two primary tumors that were synchronously identified in the ascending colon and in the rectum. A large proportion of histopathologic reports (36.3\%) did not specify the degree of differentiation of their respective tumors. Among those whose histologic grades were specified, $37.9 \%$ of tumors were well differentiated while only $6.5 \%$ were poorly differentiated. Histologic variants associated with aggressive features were noted in 39 samples, mostly of the mucinous type (Table 1).

\section{Determination of Mismatch Repair Status}

Tumors proficient in MMR proteins were identified by diffuse brownish nuclear staining with all the four antibodies against MLH1, MSH2, MSH6 and PMS2 observed under light microscopy (Figure 2). The complete absence of nuclear staining with at least one of the four MMR proteins demonstrated dMMR status (Figures 3 and 4).

Of the 77 tissues that underwent IHC staining, 61 samples (79\%) had pMMR status while 16 samples (21\%) were found to have dMMR status. Among the 16 dMMR tumors identified by IHC, seven were due to deficiency in both MSH2 and MSH6 while four were due to deficiency in both MLH1 and PMS2. Three tissue samples demonstrated the loss of PMS2 only. There were 2 unexpected cases of isolated loss of MSH2 with intact MSH6 (Figure 5). A summary of the frequencies of these staining patterns is illustrated in Figure 6.

\section{Features of $P M M R$ and $d M M R$ Tumors in Young CRC Patients}

Clinicopathologic features of the tumors with pMMR and dMMR tumors were compared (Table 2). The dMMR group had a mean age of 37.1 years, while the pMMR group had a mean age of 37.8 years. Sex distribution was relatively equal among males and females in the pMMR group ( $49.2 \%$ vs $50.8 \%$ ), but there were almost 1.3 times more females than males in the dMMR group $(56.25 \%$ vs
Table I Demographic and Clinicopathologic Data of Patients with Young-Onset Colorectal Cancer

\begin{tabular}{|c|c|c|}
\hline & $\begin{array}{l}\text { Number } \\
(n=124)\end{array}$ & Percentage \\
\hline \multicolumn{3}{|l|}{ Age } \\
\hline $0-29$ & 21 & 16.9 \\
\hline $30-39$ & 45 & 36.3 \\
\hline $40-45$ & 58 & 46.8 \\
\hline \multicolumn{3}{|l|}{ Sex } \\
\hline Female & 59 & 47.6 \\
\hline Male & 65 & 52.4 \\
\hline \multicolumn{3}{|c|}{$\begin{array}{l}\text { Number of family members with } \\
\text { cancer }\end{array}$} \\
\hline$>2$ & 9 & 7.3 \\
\hline $\mathrm{I}-2$ & 24 & 19.4 \\
\hline 0 & 89 & 71.8 \\
\hline Not reported & 2 & 1.6 \\
\hline \multicolumn{3}{|l|}{ Clinical stage } \\
\hline I & 3 & 2.4 \\
\hline II & 20 & 16.1 \\
\hline III & 58 & 46.8 \\
\hline IV & 41 & 33.1 \\
\hline Unknown & 2 & 1.6 \\
\hline \multicolumn{3}{|l|}{ Tumor location } \\
\hline Ascending colon & 24 & 19.4 \\
\hline Transverse colon & 2 & 1.6 \\
\hline Descending colon & 11 & 8.9 \\
\hline Sigmoid colon & 27 & 21.8 \\
\hline Rectum & 56 & 45.2 \\
\hline Multiple sites* & 1 & 0.8 \\
\hline Unknown & 3 & 2.4 \\
\hline \multicolumn{3}{|l|}{ Degree of differentiation } \\
\hline Well-differentiated & 47 & 37.9 \\
\hline Moderately differentiated & 24 & 19.4 \\
\hline Poorly differentiated & 8 & 6.5 \\
\hline Not specified & 45 & 36.3 \\
\hline \multicolumn{3}{|l|}{ Histologic variants } \\
\hline Mucinous & 31 & 25.0 \\
\hline Signet ring & 7 & 5.6 \\
\hline Neuroendocrine & $\mathrm{I}$ & 0.8 \\
\hline Not specified & 85 & 68.5 \\
\hline
\end{tabular}

Note: *One patient had two synchronous primary tumors (ascending colon and rectum).

43.75\%). There were more patients with stage III disease in both pMMR and dMMR groups (47.5\% and $43.75 \%$, respectively) compared to other stages. Deficient MMR tumors were most frequently located in the ascending colon, comprising $40 \%$ of cases. However, collectively, there were still more left-sided tumors compared to right- 


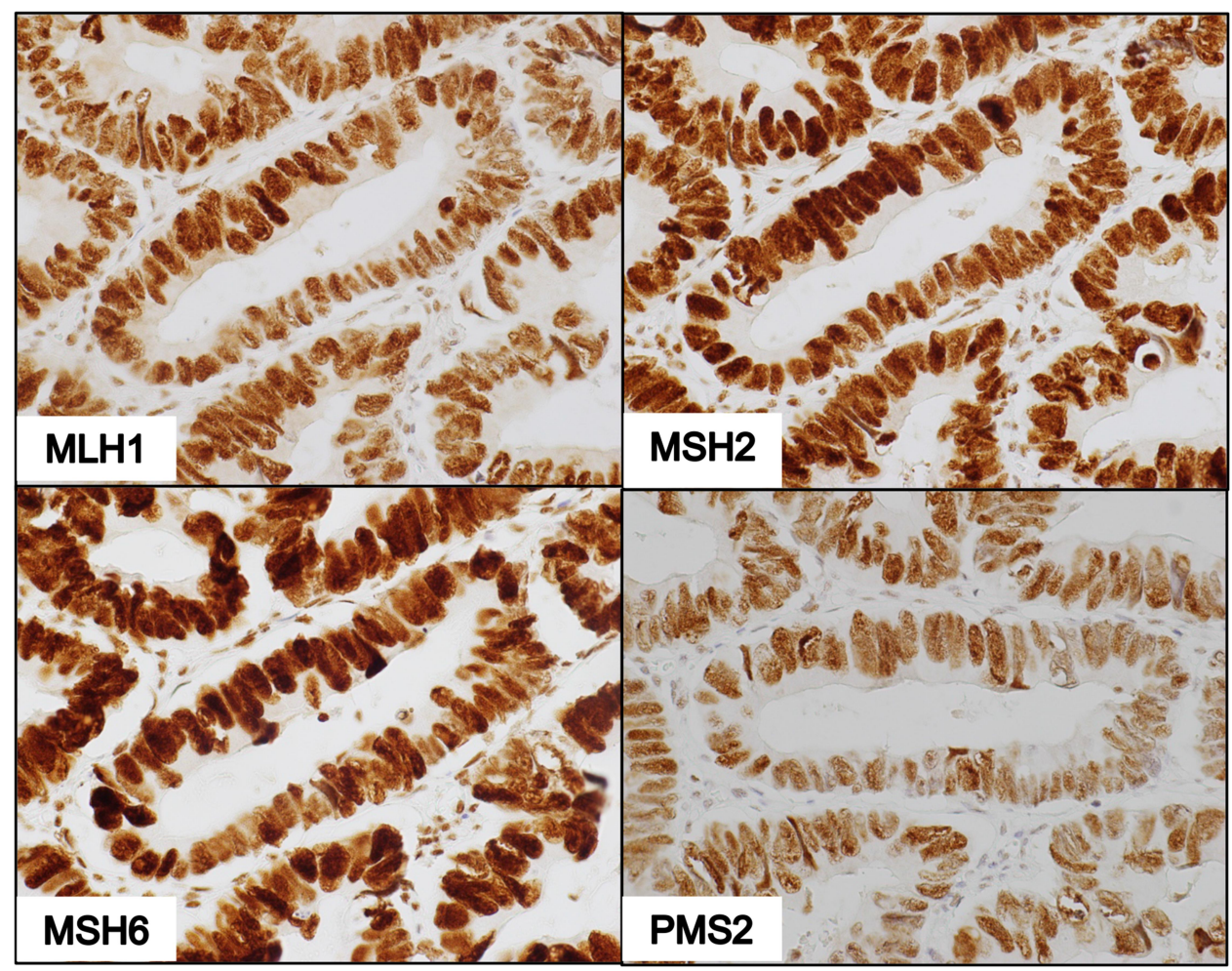

Figure $2 \mathrm{IHC}$ of PMMR tumor showing cells that form glandular structures with positive nuclear staining for antibodies against MLHI, MSH2, MSH6, and PMS2 (400X magnification).

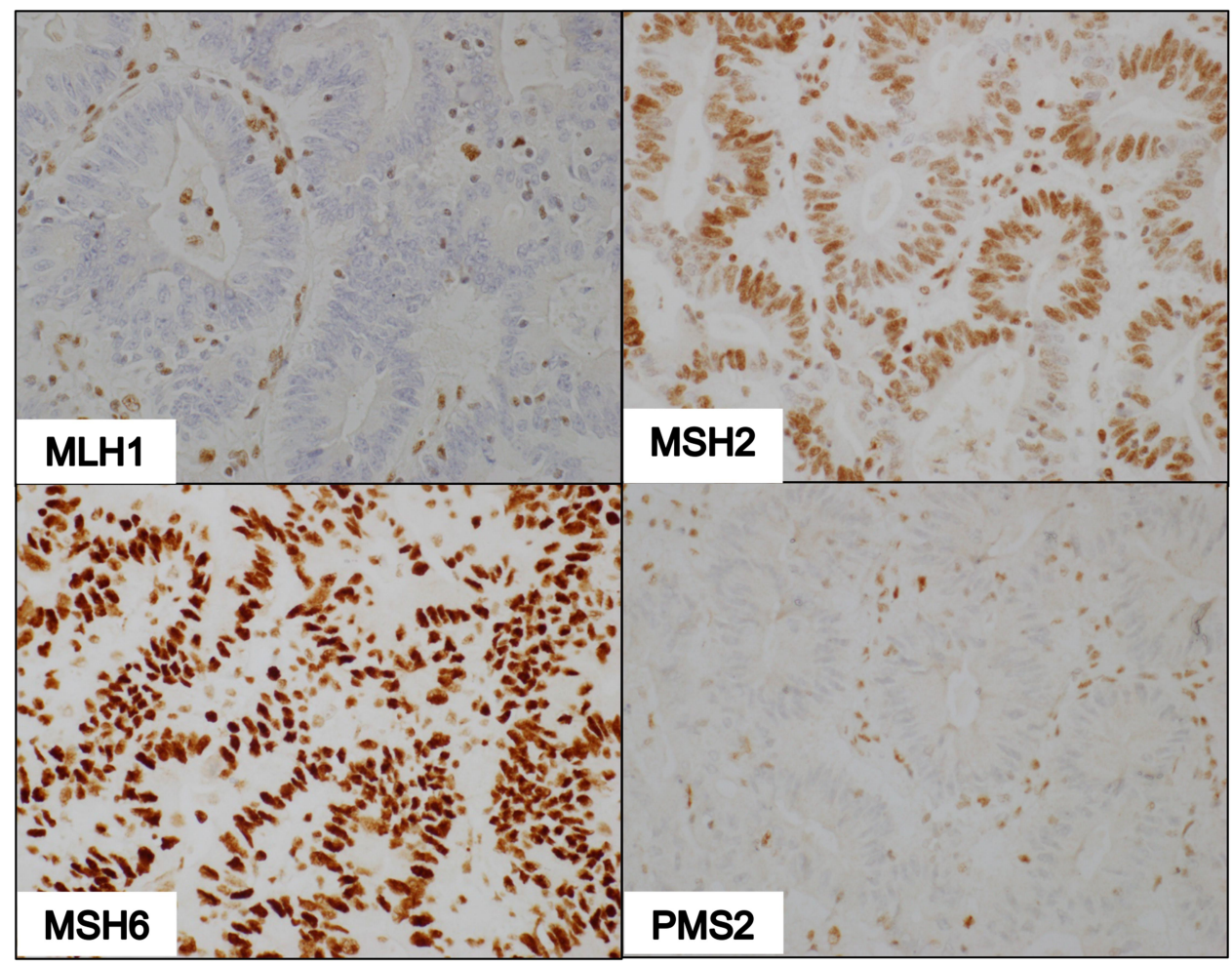

Figure $3 \mathrm{IHC}$ of $\mathrm{DMMR}$ tumor deficient in MLHI and PMS2. Photomicrographs show non-reactivity to MLHI and PMS2 antibodies, faint nuclear staining with MSH2 antibodies, and intense staining with MSH6 antibodies (100X magnification). 


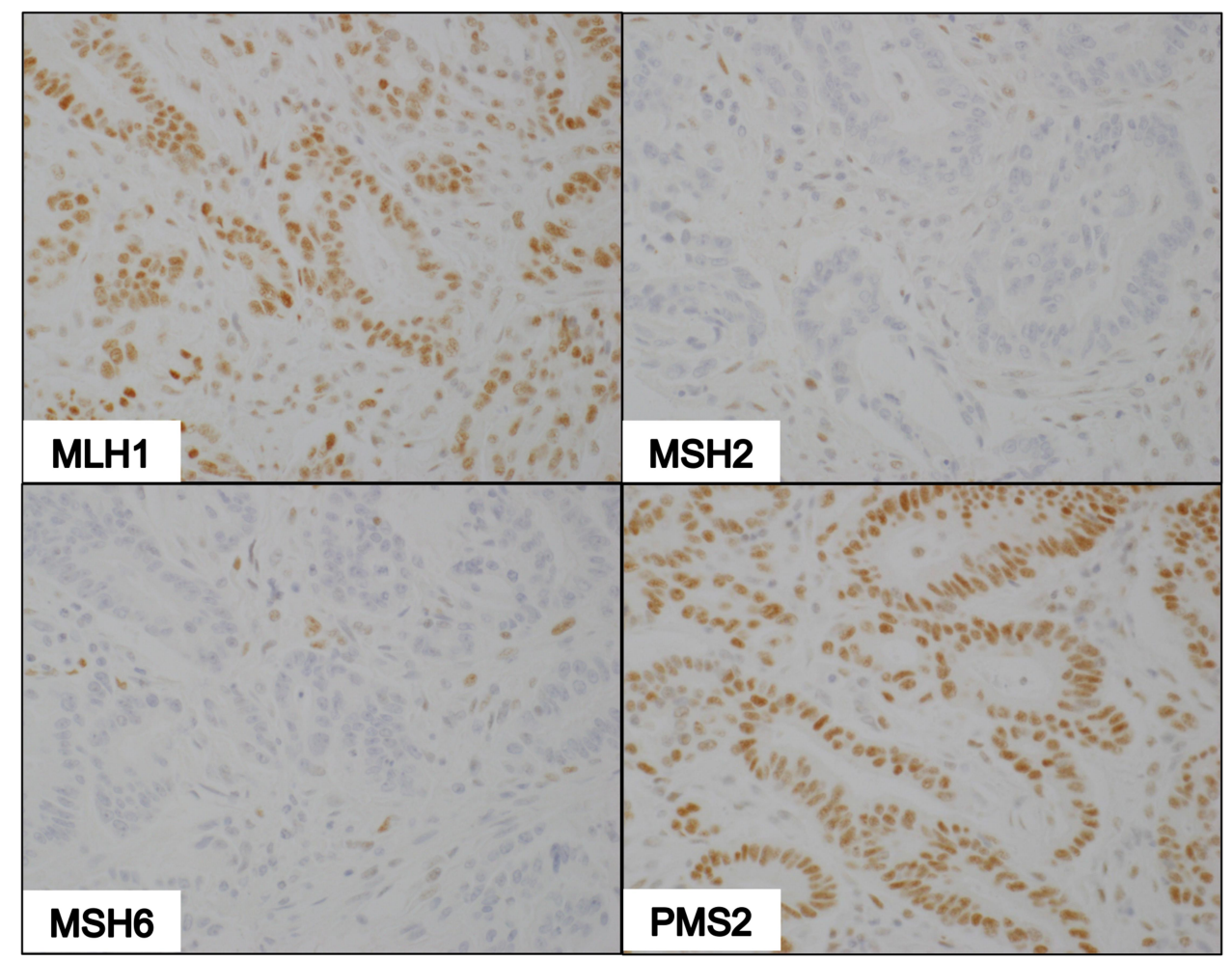

Figure $4 \mathrm{IHC}$ of dMMR tumor deficient in MSH2 and MSH6. Photomicrographs show nuclear staining with MLHI and PMS2 antibodies, and non-reactivity to MSH2 and MSH6 antibodies (I00X magnification).

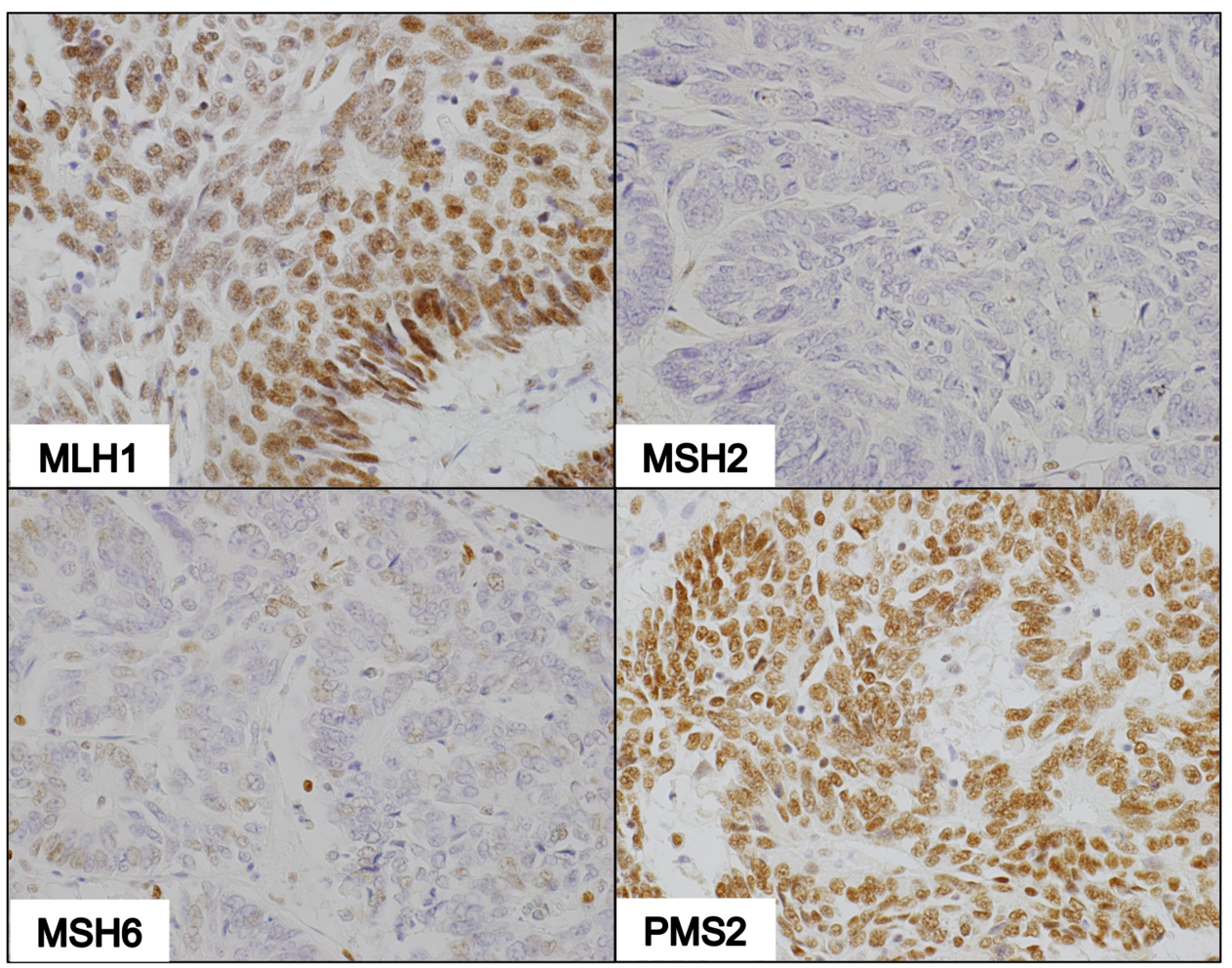

Figure $5 \mathrm{IHC}$ of dMMR tumor deficient in MSH2 only. Photomicrographs demonstrate strong nuclear staining with MLHI and PMS2, and weak staining with MSH6 in a few tumor cells in the absence of MSH2 staining (100X magnification). 


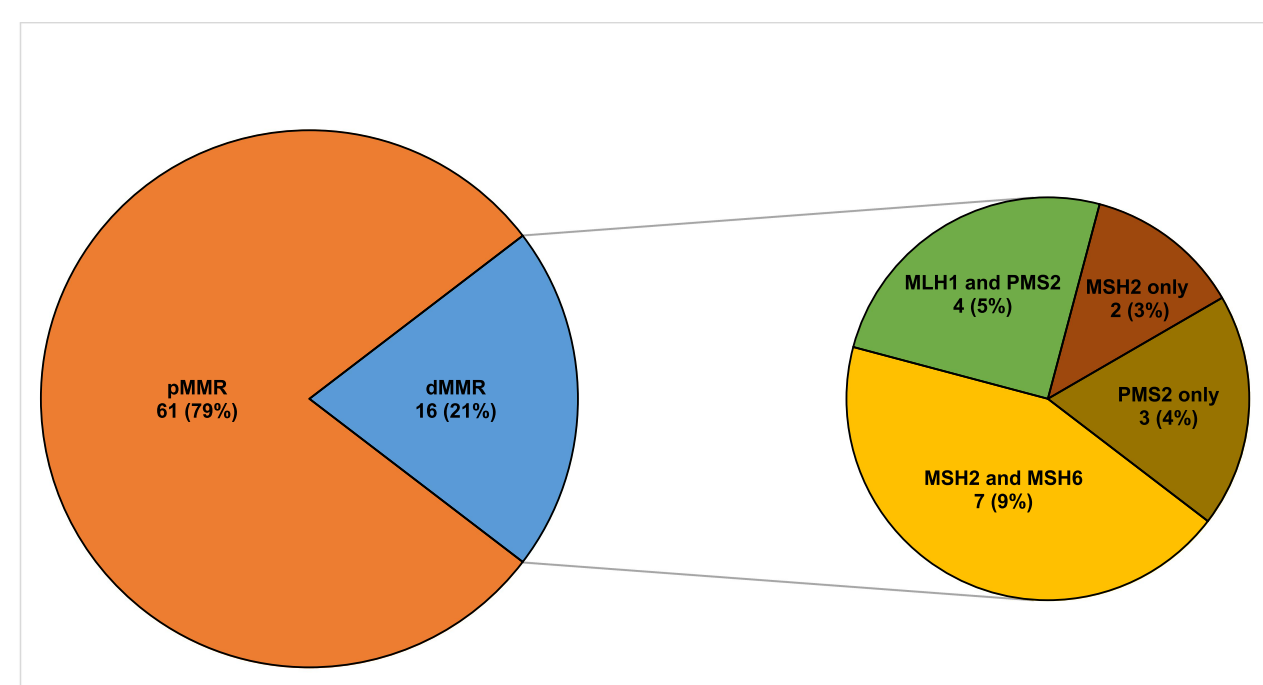

Figure 6 Mismatch repair status of colorectal tumors and types of mismatch repair deficiencies based on IHC.

Abbreviations: pMMR, proficient mismatch repair; dMMR, deficient mismatch repair; IHC, immunohistochemistry.

sided ones (9 vs 6 ) within the dMMR group. Of note, the only patient with two synchronous primary tumors had dMMR status.

There were only 3 poorly differentiated tumors seen in the pMMR group while only one was seen among dMMR tumors. Well-differentiated tumors were more common in the pMMR and dMMR groups $(44.3 \%$ and $40.0 \%$, respectively). Although patients from the 2 groups both presented with aggressive histologic variants, there was a greater proportion of these variants in the dMMR group, led by tumors with mucinous features $(50.0 \%)$.

With respect to family history, most patients from pMMR and dMMR groups denied having relatives with cancer $(75.41 \%$ and $68.75 \%$, respectively). Logistic regression analysis of the clinicopathologic features showed that older age ( $\geq 30$ years), male sex, and having metastatic disease decreased the chances of having a dMMR tumor. On the other hand, having more relatives with cancer, presence of right-sided tumors (ascending and transverse colon), and having aggressive histologic features increased the risk of a dMMR tumor. However, none of these associations were statistically significant due to the limited samples (Table 3).

\section{Discussion}

Several single-center Philippine studies have attempted to profile young CRC patients. A study by Chang et al reviewed a retrospective cohort of 73 patients with CRC diagnosed $\leq 40$ years old. Of these, 32 had colon cancer while 41 had rectal cancer. Most patients had poorly differentiated tumors. Clinical stage II and III disease were equally most prevalent in this population. ${ }^{5}$ Another study by Uy et al reported a mean age of 30.7 years for their retrospective cohort of thirty-five patients. Most of these patients presented with mucinous histology. Locally advanced disease was seen in $74.3 \%$ of this population. This study performed IHC staining to determine MMR status based only on MLH1 and MSH2 expression and found that $22.9 \%$ of young CRC cases were dMMR. ${ }^{24} \mathrm{~A}$ more recent unpublished study by Resoco et al done at the PGH reviewed the records of 270 patients with CRC diagnosed $\leq 40$ years. The mean age of patients was 35.4 years with slightly more females than males diagnosed. The rectum was the more common primary site in $56 \%$ of the patients studied. Left-sided tumors were seen in $79 \%$ of patients. Adenocarcinoma was reported to be the diagnosis in 211 cases and regardless of histology, 185 tumors were well differentiated. Eighty-five percent of patients presented with more advanced disease.

These findings were consistent with our results concerning age and site of primary tumor (left-predominant). It must be mentioned that there were fewer stage IV cases in our study because most patients who presented with metastatic disease no longer underwent curative surgical resection precluding retrieval of adequate tissue for testing. 
Table 2 Clinicopathologic Features of Young-Onset CRC Patients with pMMR and dMMR Status

\begin{tabular}{|c|c|c|}
\hline & $\begin{array}{l}\text { Number of Patients with pMMR Status } \\
\qquad \begin{array}{l}\text { (\%) } \\
n=61\end{array}\end{array}$ & $\begin{array}{l}\text { Number of Patients with dMMR Status } \\
\qquad \begin{array}{l}\text { (\%) } \\
n=16\end{array}\end{array}$ \\
\hline \multicolumn{3}{|l|}{ Age } \\
\hline $0-29$ & 7 (1 I.48) & $3(18.75)$ \\
\hline $30-39$ & $23(37.70)$ & $6(37.50)$ \\
\hline $40-49$ & $31(50.82)$ & $7(43.75)$ \\
\hline \multicolumn{3}{|l|}{ Sex } \\
\hline Female & $31(50.82)$ & $9(56.25)$ \\
\hline Male & $30(49.18)$ & $7(43.75)$ \\
\hline \multicolumn{3}{|c|}{ Number of family members with cancer } \\
\hline$>2$ & $3(4.92)$ & $2(12.50)$ \\
\hline $\mathrm{I}-2$ & $12(19.67)$ & $3(18.75)$ \\
\hline 0 & $46(75.4 I)$ & II (68.75) \\
\hline \multicolumn{3}{|l|}{ Clinical stage } \\
\hline I & I (I.64) & $2(12.50)$ \\
\hline II & $10(16.39)$ & $5(31.25)$ \\
\hline III & $29(47.54)$ & $7(43.75)$ \\
\hline IV & $20(32.79)$ & I (6.25) \\
\hline Unknown & I (I.64) & I (6.25) \\
\hline \multicolumn{3}{|c|}{ Location of primary tumor } \\
\hline \multicolumn{3}{|l|}{ Right-sided } \\
\hline Ascending colon & $8(13.11)$ & $6(37.50)$ \\
\hline Transverse colon & I (I.64) & I (6.25) \\
\hline \multicolumn{3}{|l|}{ Left-sided } \\
\hline Descending colon & $3(4.92)$ & $5(31.25)$ \\
\hline Sigmoid & $22(36.07)$ & I (6.25) \\
\hline Rectum & $26(42.62)$ & $2(12.50)$ \\
\hline Multiple sites* & $0(0.00)$ & I (6.25) \\
\hline Unknown & I (I.64) & $0(0.00)$ \\
\hline \multicolumn{3}{|c|}{$\begin{array}{l}\text { Degree of differentiation (histologic } \\
\text { grade) }\end{array}$} \\
\hline Well differentiated & $27(44.26)$ & $6(37.50)$ \\
\hline Moderately differentiated & $13(2|.3|)$ & $3(18.75)$ \\
\hline Poorly differentiated & $3(4.92)$ & I (6.25) \\
\hline Not specified & $18(29.51)$ & $6(37.50)$ \\
\hline \multicolumn{3}{|l|}{ Histologic variants } \\
\hline Mucinous & $16(26.23)$ & $8(50.00)$ \\
\hline Signet ring & $4(6.56)$ & $0(0.00)$ \\
\hline Neuroendocrine & $0(0.00)$ & I (6.25) \\
\hline Not specified & $4 I(67.2 I)$ & $7(43.75)$ \\
\hline
\end{tabular}

Note: *One patient had 2 synchronous primary tumors (ascending colon and rectum).

Data from our study contrasts with results from the US Nurse's Health Study (NHS) II that prospectively evaluated 89,278 individuals and identified 118 cases of young-onset CRC. The median age at diagnosis was 45 years (interquartile range: 41-47), which was older than that observed in our study. Here, colon cancer was more frequently diagnosed than rectal cancer. Compared to our study, this large cohort included only women. ${ }^{6}$ Our results resonate with those of NHS II and other US data that describe more advanced disease being found at diagnosis among young CRC patients regardless of MMR status. ${ }^{25}$ 
Table 3 Crude Odds Ratios and Adjusted Odds Ratios for Clinicopathologic Features in Relation to dMMR Status of Young Colorectal Cancer Patients

\begin{tabular}{|c|c|c|c|c|}
\hline Clinicopathologic Features & Crude OR & $95 \% \mathrm{Cl}$ & Adjusted OR & $95 \% \mathrm{Cl}$ \\
\hline \multicolumn{5}{|l|}{ Age } \\
\hline $0-29 *$ & & - & & - \\
\hline $30-39$ & 0.51 & $0.10-2.67$ & 0.33 & $0.05-2.19$ \\
\hline $40-49$ & 0.47 & $0.09-2.34$ & 0.30 & $0.05-1.9 \mid$ \\
\hline \multicolumn{5}{|l|}{ Sex } \\
\hline Female* & & & & - \\
\hline Male & 0.59 & $0.18-1.98$ & 0.48 & $0.10-2.39$ \\
\hline \multicolumn{5}{|l|}{ Number of family members with cancer } \\
\hline$>2$ & 1.50 & $0.14-15.96$ & 2.08 & $0.14-29.88$ \\
\hline $\mathrm{I}-2$ & 1.12 & $0.27-4.74$ & 0.88 & $0.12-6.46$ \\
\hline $0^{*}$ & & & & \\
\hline \multicolumn{5}{|l|}{ Clinical Stage } \\
\hline Non-metastatic (stages I-III)* & & & & - \\
\hline Metastatic (stage IV) & 0.17 & $0.02-1.37$ & 0.20 & $0.02-2.10$ \\
\hline \multicolumn{5}{|l|}{ Location of primary tumor } \\
\hline Right-sided (ascending and transverse) & 3.47 & $0.7 \mid-15.38$ & 2.86 & $0.60-13.48$ \\
\hline Left-sided (descending, sigmoid and rectum)* & & & & - \\
\hline \multicolumn{5}{|l|}{ Histologic variants } \\
\hline Non-aggressive* & & & & - \\
\hline Aggressive & 4.20 & $1.23-14.29$ & 2.70 & $0.62-11.86$ \\
\hline
\end{tabular}

Note: *Reference category.

Sargent et al estimated the frequency of dMMR tumors to be roughly $17 \%$ among patients with stage II/III based on the multinational ACCENT Group database. ${ }^{26}$ We report a frequency of dMMR tumors at $21 \%$ for stage II/III and $20 \%$ across all stages, but our data is limited to young-onset CRC among Filipinos.

Family history clues-in the presence of a hereditary condition such as HNPCC; but the inability to elicit a family history of cancer does not rule out HNPCC. For example, $68.75 \%$ of patients with dMMR tumors report having no relatives with cancer despite the expected association between dMMR and hereditary cancers. It is worth pointing out that our study showed that a stronger family history of cancer increases the risk for dMMR status although this finding is not statistically significant. The effect of recall and reporting bias cannot be discounted as an explanation for this discrepancy. Taking this observation into account, we believe that it is more reliable to test for MSI or MMR status to identify patients with hereditary CRC. Unfortunately, despite evidence favoring testing for MSI or MMR, it is not routinely performed in the Philippines.
Although pathologists may report histopathologic features that suggest a MSI-H phenotype, these alone are unreliable in identifying MSI-H tumors. ${ }^{20}$ Our findings show a significantly larger proportion of dMMR ascending colon tumors compared to pMMR tumors, which corroborates reports that point to right-sided tumors being associated with MSI-H. ${ }^{19}$ This supports the results of other studies on Filipinos. ${ }^{5,24}$ In contrast, a study by Lee et al conducted on Asian, mainly Chinese, immigrants to the US has shown that among HNPCC patients, left-sided tumors predominated. These observations warrant further investigation.

Loss of MLH1 and PMS2 expression is the most common dMMR IHC pattern. ${ }^{27}$ In our study, this was found to be less frequent than deficiency in both MSH2 and MSH6 although this may not be significant due to the small sample size. A similar observation was reported by Jung et al, surmising that a lower incidence of the MLH1/ PMS2-deficient IHC pattern was due to false-positive cases. This was attributed to a non-functional missense mutation in the MLH1 gene that resulted in an antigenically reactive protein. ${ }^{28}$ 
Another notable finding regarding dMMR IHC patterns is the presence of isolated MSH2 deficiency in 2 samples. This aberrant pattern has also been observed in three reports which offer no clear explanation for this uncommon result. ${ }^{28-30}$ Because degradation of MSH6 is expected in the absence of MSH2 expression, it is difficult to account for this phenomenon. One possible cause may be poor tissue preparation or staining technique that could have led to non-specific binding of MSH6 antibodies or to non-binding of MSH2 antibodies to their target protein. Very faint or patchy IHC staining may also lead to subjective misinterpretation of the results although the samples in this study were thoroughly reviewed by our pathologist.

Our study has several limitations. First, this study involves patients from two hospitals within the National Capital Region, which may inadequately represent the population of young Filipinos with CRC. Second, variability in the reporting of histopathologic findings between samples in the study due to differences in reporting standards between surgical pathology laboratories hampered comparison of pMMR and dMMR tumors using histopathologic features such as degree of differentiation of tumor cells. Third, the limited number of specimens obtained from patients due to metastatic disease affected the estimates of the frequencies reported for MMR status. This also explains why it is difficult to make generalizations about the larger population of CRC patients based on our findings due to non-significant results on statistical analysis.

Nevertheless, this is the first study to profile MMR status among young Filipinos with CRC. It is hoped that larger prospective studies will profile a broader population of Filipinos in order to better estimate the true prevalence of dMMR and pMMR among young CRC patients.

\section{Conclusion}

This is the first attempt to profile young-onset Filipino CRC patients according to MMR status. IHC of tumor samples can easily identify the MMR status of a patient and can serve as a substitute for molecular testing to determine MSI status. This inexpensive technique needs to be part of standard evaluation of colorectal cancer because of its utility in clinical decision-making.

\section{Acknowledgments}

This study was supported by grants from the University of the Philippines System (OVPAA-EIDR Code 06-008) and the Philippine Council for Health Research and Development (grant code FP150025). The authors thank Prof. Cynthia P. Cordero of the Clinical Epidemiology Unit, University of the Philippines College of Medicine for her contribution in the statistical analysis of the study data.

\section{Disclosure}

The authors report no conflicts of interest in this work.

\section{References}

1. Ferlay J, Soerjomataram I, Ervik M, et al., Cancer Incidence and Mortality Worldwide: IARC CancerBase No. 11. International Agency for Research on Cancer; Lyon, France: 2013. Available from: http://globocan.iarc.fr;. Accessed February 18, 2021.

2. Laudico AV, Medina V, Mirasol-Lumague MR, et al. 2010 Philippine Cancer Facts and Estimates. Manila: Philippine Cancer Society, Inc; 2010.

3. Laudico AV, Mirasol-Lumague MR, Medina V, Mapua CA, Valenzuela FG, Pukkala E. 2015 Philippine Cancer Facts and Estimates. Manila: Philippine Cancer Society, Inc; 2015.

4. Kaw LL, Punzalan CK, Crisostomo AC, Bowyer MW, Wherry DC. Surgical pathology of colorectal cancer in Filipinos: implications for clinical practice. J Am Coll Surg. 2002;195:188-195. doi:10.1016/ S1072-7515(02)01186-9

5. Chang RL, Roxas MF, Asprer JM. Colorectal cancer in the young: a five-year review of cases. Philipp J Surg Spec. 2003;58(1):32-35.

6. Nguyen LH, Liu PH, Zheng X, et al. Sedentary behaviors, TV viewing time, and risk of young-onset colorectal cancer. JNCI Cancer Spectr. 2019;2:pky073. doi:10.1093/jncics/pky073

7. Grady WM. CIMP and colon cancer gets more complicated. Gut. 2007;56(11):1498-1500. doi:10.1136/gut.2007.125732

8. Ogino S, Goel A. Molecular classification and correlates in colorectal cancer. J Mol Diagn. 2008;10:13-27. doi:10.2353/jmoldx.200 8.070082

9. Hashmi AA, Ali R, Hussain ZF, et al. Mismatch repair deficiency screening in colorectal carcinoma by a four-antibody immunohistochemical panel in Pakistani population and its correlation with histopathological parameters. World J Surg Oncol. 2017;15:116. doi:10.1186/s12957-017-1158-8

10. Kawakami H, Zaanan A, Sinicrope FA. Microsatellite instability testing and its role in the management of colorectal cancer. Curr Treat Options Oncol. 2015;16:30. doi:10.1007/s11864-015-0348-2

11. Smith C, Butler JA. Colorectal cancer in patients younger than 40 years of age. Dis Colon Rectum. 1989;32:843-846. doi:10.1007/ BF02554552

12. De la Chapelle A, Hampel H. Clinical relevance of microsatellite instability in colorectal cancer. J Clin Oncol. 2010;28:3380-3387. doi:10.1200/JCO.2009.27.0652

13. Loughrey MB, McGrath J, Coleman HG, et al. Identifying mismatch repair-deficient colon cancer: near-perfect concordance between immunohistochemistry and microsatellite instability testing in a large, population-based series. Histopathology. 2020. doi:10.1111/his.14233

14. Li GM. Mechanisms and functions of DNA mismatch repair. Cell Res. 2008;18:85-98. doi:10.1038/cr.2007.115

15. Hall G, Clarkson A, Shi A, et al. Immunohistochemistry for PMS2 and MSH6 alone can replace a four antibody panel for mismatch repair deficiency screening in colorectal adenocarcinoma. Pathology. 2010;42:409-413.

16. Gatalica Z, Vranic S, Xiu J, Swensen J, Reddy S. High microsatellite instability (MSI-H) colorectal carcinoma: a brief review of predictive biomarkers in the era of personalized medicine. Fam Cancer. 2016;15:405-412. doi:10.1007/s10689-016-9884-6 
17. Greenson JK, Huang SC, Herron C, et al. Pathologic predictors of microsatellite instability in colorectal cancer. Am J Surg Pathol. 2009;33:126-133. doi:10.1097/PAS.0b013e31817ec2b1

18. Lee J, Xiao YY, Sun YY, et al. Prevalence and characteristics of hereditary non-polyposis colorectal cancer (HNPCC) syndrome in immigrant Asian colorectal cancer patients. BMC Cancer. 2017;17:843. doi:10.1186/s12885-017-3799-y

19. Baran B, Ozupek NM, Tetik NY, Acar E, Bekcioglu O, Baskin Y. Difference between left-sided and right-sided colorectal cancer: a focused review of literature. Gastroenterol Res. 2018;11:264-273.

20. Alexander J, Watanabe T, Wu TT, Rashid A, Li S, Hamilton SR. Histopathological identification of colon cancer with microsatellite instability. Am J Pathol. 2001;158:527-535. doi:10.1016/S0002-9440(10)63994-6

21. Overman MJ, Lonardi S, Wong KY, et al. Durable clinical benefit with nivolumab plus ipilimumab in DNA mismatch repair-deficient/ microsatellite instability-high metastatic colorectal cancer. J Clin Oncol. 2018;36:773-779. doi:10.1200/JCO.2017.76.9901

22. Le DT, Uram JN, Wang H, et al. PD-1 blockade in tumors with mismatch-repair deficiency. $N$ Engl J Med. 2015;372:2509-2520. doi:10.1056/NEJMoa1500596

23. Stigliano V, Sanchez-Mete L, Martayan A, Anti M. Early-onset colorectal cancer: a sporadic or inherited disease? World J Gastroenterol. 2014;2014(20):12420. doi:10.3748/wjg.v20.i35.12420

24. Uy GB, Kaw LL, Punzalan CK, et al. Clinical and molecular biologic characteristics of early-onset versus late-onset colorectal carcinoma in Filipinos. World J Surg. 2004;28:117-123. doi:10.1007/s00268-003-7281-4
25. Dozois EJ, Boardman LA, Suwanthanma W, et al. Young-onset colorectal cancer in patients with no known genetic predisposition: can we increase early recognition and improve outcome? Medicine. 2008;87:259. doi:10.1097/MD.0b013e3181881354

26. Sargent DJ, Shi Q, Yothers G, et al. Prognostic impact of deficient mismatch repair (dMMR) in 7,803 stage II/III colon cancer (CC) patients (pts): a pooled individual patient data analysis of 17 adjuvant trials in the ACCENT database. J Clin Oncol. 2014;32:5s. doi:10.1200/jco.2014.32.15_suppl.3507

27. Lee CT, Chow NH, Chen YL, et al. Clinicopathological features of mismatch repair protein expression patterns in colorectal cancer. Pathol Res Pract. 2020;217:153288. doi:10.1016/j.prp.2020.153288

28. Jung J, Kang Y, Lee YJ, et al. Comparison of the mismatch repair system between primary and metastatic colorectal cancers using immunohistochemistry. J Pathol Transl Med. 2017;51:129-136. doi:10.4132/jptm.2016.12.09

29. Pearlman R, Markow M, Knight D, et al. Two-stain immunohistochemical screening for Lynch syndrome in colorectal cancer may fail to detect mismatch repair deficiency. Mod Pathol. 2018;31:1891-1900.

30. Roth RM, Haraldsdottir S, Hampel H, Arnold CA, Frankel WL. Discordant mismatch repair protein immunoreactivity in Lynch syndrome-associated neoplasms: a recommendation for screening synchronous/metachronous neoplasms. Am J Clin Pathol. 2016;146:50 56. doi:10.1093/ajcp/aqw067

\section{Publish your work in this journal}

Cancer Management and Research is an international, peer-reviewed open access journal focusing on cancer research and the optimal use of preventative and integrated treatment interventions to achieve improved outcomes, enhanced survival and quality of life for the cancer patient.
The manuscript management system is completely online and includes a very quick and fair peer-review system, which is all easy to use. Visit http://www.dovepress.com/testimonials.php to read real quotes from published authors. 